\title{
Non-linear Analysis of Vibrating Flip-flow Screens
}

\author{
Sanpeng Gong ${ }^{1,2}$, Xinwen Wang ${ }^{1}$ and Sebastian Oberst ${ }^{2}$ \\ 1 School of Chemical and Environmental Engineering, China University of Mining and Technology, Beijing, China \\ ${ }^{2}$ Centre for Audio, Acoustics and Vibration, School of Software, University of Technology Sydney, Australia
}

\begin{abstract}
Vibrating flip-flow screens provide an effective solution for the screening of highly viscous or fine materials. Apart from other factors, the vibration characteristics of the main and floating screen frames are largely responsible for the flip-flow screen's sifting performance and its processing capacity. In this paper, the vibration characteristics of a vibrating flip-flow screen with linear and nonlinear springs are compared. Analytical results highlight that increasing the relative amplitude and avoiding undesirable resonances of the main and the floating screen frames can be realised to improve the screen's performance. The materials on the screen panel have less an effect on the vibration characteristics of the vibrating flip-flow screen with nonlinear springs than using linear springs. Other design parameters which influence the performance of vibrating flip-flow screens are discussed.
\end{abstract}

\section{Introduction}

Dry screening of wet and fine materials is an on-going issue in processing minerals and ore such as gold, iron or coal. During traditional screening equipment frequency experience the problem of plugging, which is a blockage of the screen's grid with debris [1-2]. Vibrating flip-flow screens (VFFS) are used as machinery to tackle the problem of sifting the materials. A VFFS has elastic screen panels which are stretched and relaxed, thereby creating changing tension motion to efficiently sort loose materials. VFFS are widely used in screening processes of fine materials where it is superior to conventional vibrating screens [3-4].

Common influencing factors on the screening performance of VFFS include the rotational speed of the exciter, the inclination angle of the screen, the slack length of the screening panel among many other factors related to material itself. Derived from e.g. the rotational speed are the vibration characteristics of VFFS, which also largely affect the processing performance and capacity [5-6]. To achieve the best screen performance, an optimized design and control is required, which fundamentally needs an indepth understanding of the vibrations.

Xiong [6] analysed the dynamics of a banana flip-flow screen with linear springs. However, in the actual situation, vibrating flip-flow screens with linear springs can't work at the ideal working area as the change of the system' natural frequency caused by material quantity (additional mass) or changing stiffness (pre-tension, tension). The need to design vibrating flip-flow screens which are efficient over a broad band of forcing frequencies led to the study nonlinear characteristics - considering nonlinear stiffness. This however, requires developing an analytical procedure, to study the system's steady state responses and stability behaviour which we are doing in the following.

\section{Analytical model}

The investigated VFFS is shown schematically in Fig.1(a). The main screen frame is supported by vibration isolation springs. The inserted floating screen frame is connected to the main screen frame by shear springs. Moreover, the VFFS is excited by an exciter shaft mounted to the main frame. The rotation of the eccentric blocks in the exciter causes a relative movement between the main and the floating frame, which harmonically slackens and stretches the screen panel.

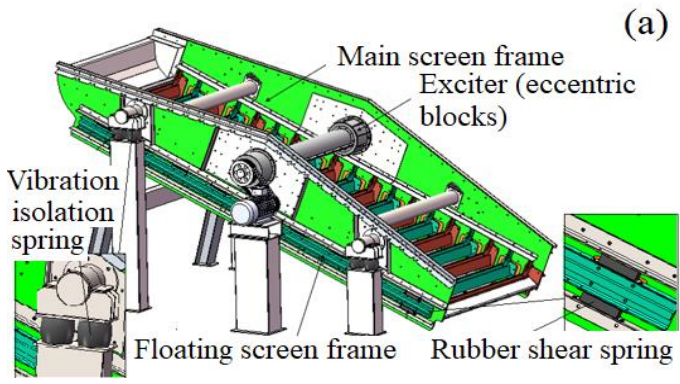




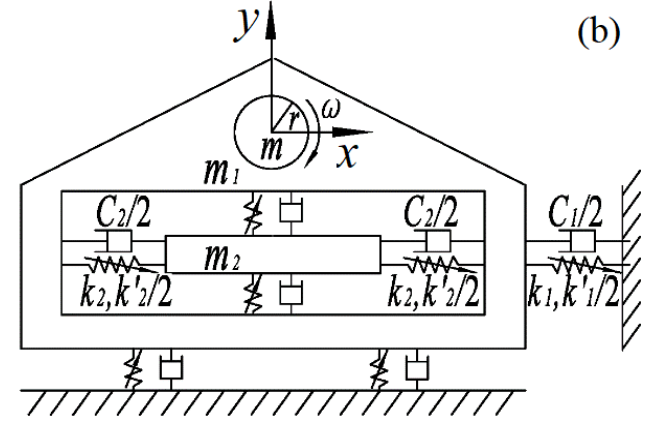

Figure 1. (a) Schematic and the (b) mathematical model of a vibrating flip-flow screen.

To investigate the dynamic of the vibrating flip-flow screen, a corresponding dynamic model is established, Fig.1(b). Here, $m$ is the eccentric block mass of vibration exciter; $m_{1}$ and $m_{2}$ are the mass of main and floating screen frames respectively, $r$ is the eccentricity; $\omega$ the angular velocity of $m ; k_{1}, k_{1}^{\prime}$ and $c_{1}$ - stiffness and damping coefficient of the vibration isolation springs, $k_{2}, k_{2}^{\prime}$ and $c_{2}-$ stiffness and damping coefficients of the vibration shear spring. The $x$-axis is along the screen panel, the $y$-axis is perpendicular to the screen panel. The co-ordinate $x_{1}$ represents the displacement of the main screen fame's mass $m_{1}$ with respect to its foundation, while $x_{2}$ stands for the relative displacement of the floating screen frame's mass $m_{2}$ with respect to the mass $m_{1}$. The vibration of the vibrating flip-flow screen in $y$ direction can be neglected, because that the main and floating screen frames will vibrate synchronously in $y$ direction when VFFS works, which cause little effect on the performance of the VFFS. Therefore, in this paper, the dynamics of the VFFS is studied only considering $x$ direction. The machine is excited by a harmonic forcing of the form $F=F_{0} \cos \omega t$ $\left(F_{0}=m r \omega^{2}\right)$. The non-linear springs restoring forces and governing equations are written as [7-8]:

$f_{i}\left(x_{i}\right)=k_{i} x_{i}+k_{i}{ }^{\prime} x_{i}{ }^{3}$, with $i=1,2$, and

$\left(m_{1}+m_{2}\right) \ddot{x}_{1}+m_{2} \ddot{x}_{2}+c_{1} \dot{x}_{1}+k_{1} x_{1}+k_{1}{ }^{\prime} x_{1}{ }^{3}=$

$F_{0} \cos \omega t ;$ and

$m_{2} \ddot{x}_{1}+m_{2} \ddot{x}_{2}+c_{2} \dot{x}_{2}+k_{2} x_{2}+k_{2}{ }^{\prime} x_{2}{ }^{3}=0$.

Suppose that the characteristic length is described by $x_{c}=$ $F_{0} / k_{1}$, then the displacements can be normalized using $x_{c}$, $y_{i}=x_{i} / x_{c}$ and the following dimensionless parameters are introduced:

$\tau=\omega t, \mu=m_{2} / m_{1}, \bar{\omega}_{i}^{2}=k_{i} / m_{i}, \omega_{i}=\bar{\omega}_{i} / \omega, \eta_{i}=$ $\omega_{i}{ }^{2}, \zeta_{i}=c_{i} / 2 \sqrt{k_{i} m_{i}}, \quad \varepsilon_{i}=2 \zeta_{i} \omega_{i}, \quad \delta=\omega_{2} / \omega_{1}, \quad \Omega=$ $\omega / \bar{\omega}_{1}, \quad \bar{\alpha}_{1}=k_{1}^{\prime} k_{1} x_{c}^{2} / m_{1}{ }^{2} \omega^{4}, \bar{\alpha}_{2}=k_{2}^{\prime} k_{2} x_{c}^{2} / m_{1} m_{2} \omega^{4}$, $\alpha_{i}=\bar{\alpha}_{i} \Omega^{2}, \bar{\lambda}=F_{0} / m_{1} \bar{\omega}_{1}{ }^{2} x_{c}$, and $\lambda=\bar{\lambda} \omega_{1}{ }^{2}$.

Important here are the dimensionless parameters $\bar{\alpha}_{1}, \bar{\alpha}_{2}$ and $\mu$ as they represent non-linear stiffness and mass ratio of $m_{2}$ and $m_{1}$ which we are going to vary in the following. The equations of motion of the system can be put in matrix form:

$\mathbf{M} \ddot{\mathbf{Y}}+\mathbf{C} \dot{\mathbf{Y}}+\mathbf{K Y}=\mathbf{F}$,

with

$$
\begin{gathered}
\mathbf{Y}=\left[\begin{array}{l}
y_{1} \\
y_{2}
\end{array}\right], \mathbf{M}=\left[\begin{array}{rr}
1+\mu & \mu \\
\mu & \mu
\end{array}\right], \mathbf{C}=\left[\begin{array}{cc}
\varepsilon_{1} & 0 \\
0 & \mu \varepsilon_{2}
\end{array}\right], \mathbf{K}= \\
{\left[\begin{array}{cc}
\eta_{1} & 0 \\
0 & \eta_{2}
\end{array}\right], \mathbf{F}=\left[\begin{array}{c}
\lambda \cos \tau-\alpha_{1} y_{1}{ }^{3} \\
-\mu \alpha_{2} y_{2}{ }^{3}
\end{array}\right] .}
\end{gathered}
$$

Applying the method of averaging [9-10] the steady state responses become of the form

$\mathbf{Y}=\mathbf{U} \cos \tau+\sin \tau$, and

$\dot{\mathbf{Y}}(\boldsymbol{\tau})=-\mathbf{U} \sin \tau+\mathbf{V} \cos \tau$

with the time dependence of $\mathbf{U}=\left[u_{1}(\tau), u_{2}(\tau)\right]^{T}$ and $\mathbf{V}=\left[v_{1}(\tau), v_{2}(\tau)\right]^{T}$ being assumed to be "slow" and of higher order. Differentiating the Eq. (4a) with respect to the time $\tau$, we obtain

$\dot{\mathbf{Y}}=\dot{\mathbf{U}} \cos \tau-\mathbf{U} \sin \tau+\dot{\mathbf{V}} \sin \tau+\mathbf{V} \cos \tau$

Substituting Eq. (4b) into Eq. (5), one finds:

$\dot{\mathbf{U}} \cos \tau+\dot{\mathbf{V}} \sin \tau=0$.

Also differentiating the Eq. (4b), we obtain

$$
\ddot{\mathbf{Y}}=-\dot{\mathbf{U}} \sin \tau-\mathbf{U} \cos \tau+\dot{\mathbf{V}} \cos \tau-\mathbf{V} \sin \tau
$$

Substituting the equations about $\mathbf{Y}, \dot{\mathbf{Y}}$ and $\ddot{\mathbf{Y}}$ into Eq. (3), the resulting equation becomes

$(\mathbf{M} \dot{\mathbf{V}}-\mathbf{M U}+\mathbf{C V}+\mathbf{K U}) \cos \tau-(\mathbf{M U}+\mathbf{M V}+\mathbf{C U}-$

$\mathbf{K V}) \sin \tau=\mathbf{F}(\boldsymbol{u}, \boldsymbol{v}, \boldsymbol{\tau})$

Then, multiplying Eq. (6) by the term $\mathbf{M} \cos \tau$, Eq. (7) is multiplied by $-\sin \tau$ and adding the two equations. The resulting equation is then integrated from 0 to $2 \pi$ by assuming that $\mathbf{U}$ and $\mathbf{V}$ remain constant. The final outcome of these manipulations is

$\mathbf{M U}=\frac{1}{2}(\mathbf{K}-\mathbf{M}) \mathbf{V}-\frac{1}{2} \mathbf{C U}+\frac{1}{2}\left(\begin{array}{c}\frac{3}{4} \alpha_{1} v_{1}\left(u_{1}^{2}+v_{1}^{2}\right) \\ \frac{3}{4} \mu \alpha_{2} v_{2}\left(u_{2}^{2}+v_{2}^{2}\right)\end{array}\right)$

Similarly, multiplying Eqs. (6) and (7) by either $\mathbf{M} \sin \tau$ or $\cos \tau$, respectively; adding both equations and integrating it from 0 to $2 \pi$, we obtain

$\mathbf{M V}=\frac{1}{2}(\mathbf{M}-\mathbf{K}) \mathbf{V}-\frac{1}{2} \mathbf{C V}+\frac{1}{2}\left(\begin{array}{c}\lambda-\frac{3}{4} \alpha_{1} u_{1}\left(u_{1}^{2}+v_{1}^{2}\right) \\ -\frac{3}{4} \mu \alpha_{2} u_{2}\left(u_{2}^{2}+v_{2}^{2}\right)\end{array}\right)$

Eqs. (8) and (9) represent a system of first order, ordinary differential equations. For periodic steady vibration, the conditions are provided as

$\dot{\mathbf{U}}=\dot{\mathbf{V}}=\mathbf{0}$.

Substituting conditions (10) into Eqs. (8) and (9), a set of four coupled non-linear algebraic equations for $u_{1}$, $v_{1}, u_{2}$ and $v_{2}$ is obtained,

$$
\begin{array}{r}
\left(\eta_{1}-\mu-1\right) v_{1}-\mu v_{2}-\varepsilon_{1} u_{1}+\frac{3}{4} \alpha_{1} v_{1}\left(u_{1}^{2}+v_{1}^{2}\right)=0, \\
-\mu v_{1}+\left(\mu \eta_{2}-\mu\right) v_{2}-\mu \varepsilon_{2} u_{2}+\frac{3}{4} \mu \alpha_{2} v_{2}\left(u_{2}^{2}+v_{2}^{2}\right)=0, \\
\left(1+\mu-\eta_{1}\right) u_{1}-\mu u_{2}-\varepsilon_{1} v_{1}+\lambda-\frac{3}{4} \alpha_{1} u_{1}\left(u_{1}^{2}+v_{1}^{2}\right)= \\
0, \text { and } \quad(11 \mathrm{c}) \\
\mu u_{1}+\left(\mu-\mu \eta_{2}\right) u_{2}-\mu \varepsilon_{2} v_{2}-\frac{3}{4} \mu \alpha_{2} u_{2}\left(u_{2}^{2}+v_{2}^{2}\right)=0 .
\end{array}
$$

\section{Numerical results and discussion}

In all calculations, the non-dimensionless frequency $\Omega$ is taken as the independent variable. $x_{1}$ and $x_{2}$ denote the response amplitudes of the main screen frame and the relative displacement of main screen frame and floating screen frame. Both $x_{1}$ and $x_{2}$ can be expressed in terms of displacement and velocity using.

$x_{i}=x_{c} \sqrt{u_{i}^{2}+v_{i}^{2}}$, with $i=1,2 \quad$ (12a)

As spring stiffness and materials have greater effect on the performance of VFFS compared with other factors. Results are obtained by applying the above analysis, calculating the effect of nonlinear spring stiffness and the 
effect of the material on the steady state response of the system.

\subsection{The effect of non-linear stiffness on the steady state response}

Several groups of parameters are tried out to discuss the vibration characteristics of the system; the selection of the relative parameters is taken by an industrial flip-flow screen $\left(m_{1}=4,482 \mathrm{~kg}, k_{1}=756.7 \mathrm{kN} / \mathrm{m}\right)$. The steady state response of the main screen frame $\left(x_{1}\right)$ and relative displacement between the main and the floating screen frame $\left(x_{2}\right)$ are shown in Figs. 2(a) and 2(b) considering different $\bar{\alpha}_{1}$ and $\bar{\alpha}_{2}$, which represent nonlinear stiffness of isolation vibration springs and shear springs, respectively.
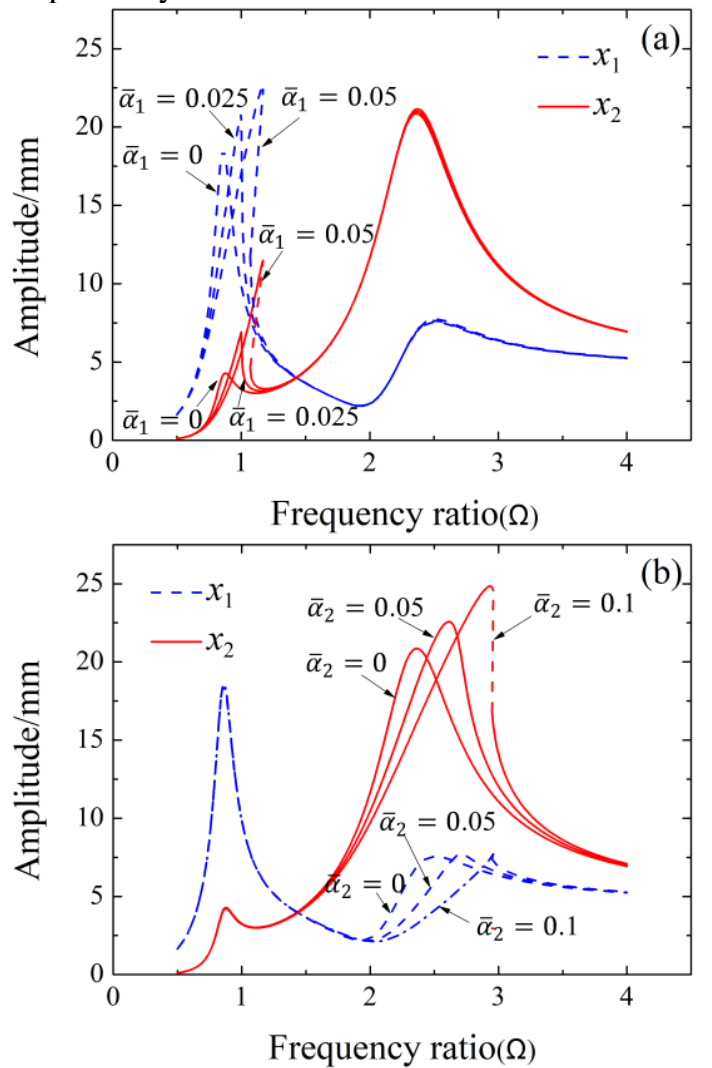

Figure 2. Steady-state response of the system: (a) $u=$ $0.3, \delta=2, \bar{\alpha}_{2}=0, \zeta_{1}=\zeta_{2}=0.1$ (b) $u=0.3, \delta=2, \bar{\alpha}_{1}=$ $0, \zeta_{1}=\zeta_{2}=0.1$

In Fig. 2(a), with the increase of the non-linear spring stiffness $\bar{\alpha}_{1}$ from zero to 0.05 in the first order resonance region, the peak values of $x_{1}$ and $x_{2}$ both increase, and the corresponding $\Omega$ of peak value also increases. This will cause an undesirable vibration mode in case the VFFS begins to work. In Fig. 2(b), increasing the non-linear spring stiffness $\bar{\alpha}_{2}$ from zero to 0.1 the peak value of $x_{2}$ increases from 20.85 to 24.58 , and the peak value of $x_{1}$ remains almost constant. Because VFFS commonly work near the second resonance region, it needs to be tested whether the increase of $\bar{\alpha}_{2}$ could be beneficial to the performance and processing capacity of materials.

\subsection{The effect of materials on vibration characteristic of the system}

When the screening panel is loaded with material $(2,045$ $\mathrm{kg}$ ) the mass-ratio changes from $\mu=0.3$ to $\mu=0.38$. The steady state responses of the VFFS with linear $\left(\bar{\alpha}_{2}=0\right)$ and nonlinear $\left(\bar{\alpha}_{2}=0.1\right)$ shear springs with and without material are shown in Figs. 3(a) and 3(b), respectively. In Fig. 3(a) it can be seen that the presence of materials has a large effect on the vibration characteristics of VFFS with linear shear spring; the large changes in $x_{1}$ and $x_{1}$ influences the stability of the system and requires to be monitored. Fig. 3(b) shows that an additional loading of material, which acts on the screen has a rather small effect on the nonlinear vibration characteristics when it works near the resonance area hence non-linear characteristics of shear springs could be beneficial to the stability of the system.

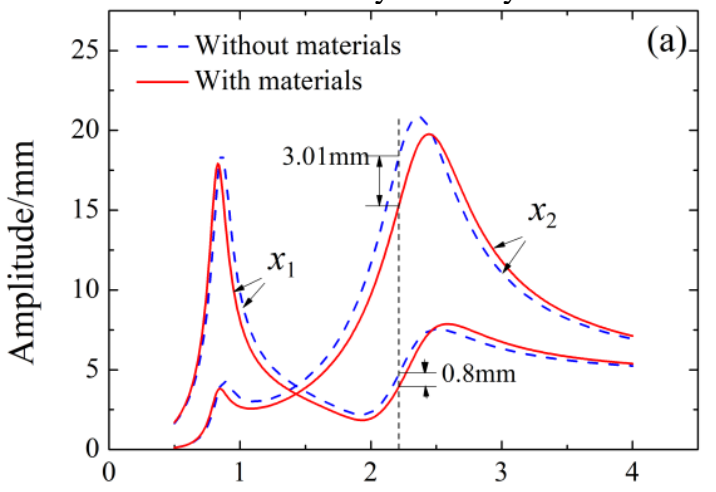

Frequency ratio $(\Omega)$

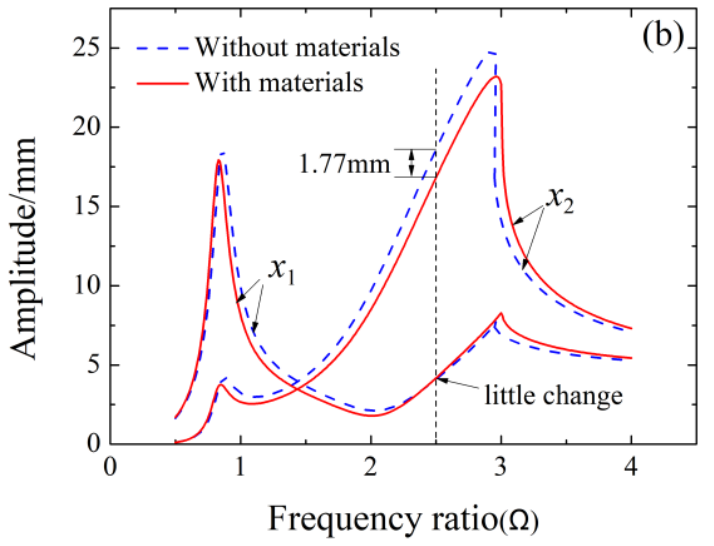

Figure. 3 Steady-state response of the system: (a) linear, and (b) nonlinear shear springs

\section{Conclusion}

Vibrating flip-flow screens can be effective solutions for the screening of highly viscous and fine materials. Our study employs an analytical procedure to determine the dynamic response of vibrating flip-flow screens by also considering their nonlinear shear spring characteristics. This analysis is applied to two cases, with the goal of identifying the effect of the system parameters on the steady-state responses. Results indicate that nonlinear springs in connection with properly system parameters would be beneficial for the vibrating flip-flow screens' performance. However, while the application of nonlinear springs could show to be useful over a wider range of forcing frequencies, the extension to a larger frequency band also requires to study whether stability issue can arise. 
Further, a comparison with real experiments and an updated finite element model would be required in the future.

\section{References}

[1] Noble A and Luttrell G 2015 Int. J. Min. Sci. Technol. 25 511-521

[2] Zheng G, Zhu J, Xia W and Liu S 2016 Filtr. + Separation. 53 38-41

[3] Akbari H, Ackah L and Mohanty M 2017 Int. J. Coal Prep. Util. 1-23
[4] Guo Q and Gong G 2016 XVIII Int. Coal Prep. Cong. (Saint- Petersburg: Springer International Publishing Switzerland) P 913-918

[5] Peng L, Liu C, Hong H, Li J and Xia Y 2014 J. China Coal Soc. 39 976-980

[6] Xiong X, Niu L, Gu C and Wang Y 2017 J. Sound Vib. 411 108-128

[7] Zhang Z, Oberst S and Lai J 2016 J. Sound Vib. 377 123-132

[8] Beltrán-Carbajal and Silva-Navarro G 2014 J. Sound Vib. 333 3019-3030

[9] Zhu S, Zheng Y and Fu Y 2004 J. Sound Vib. 271 1524

[10]Natsiavas S 1992 J. Sound Vib. 156 227-245. 